\title{
Neither Goethe nor Bismarck: on the Link between Theory and Empirics in Council Decision-Making Studies
}

\author{
Gerald Schneider
}

\author{
Experiment. \\ Make it your motto day and night. \\ Experiment \\ And it will lead you to the light.
}

Cole Porter 'Experiment' (1933)

\section{Introduction ${ }^{1}$}

In a spirited attack, Dorothée Heisenberg takes issue with the emerging field of quantitatively-oriented European Union studies in general and the formal modelling tradition in the exploration of Council of Minister decisionmaking in particular. Drawing on her important article on the 'culture of consensus' (Heisenberg 2005), she argues in Chapter 14 above that rationalist scholarship is incapable of enlightening the wheeling and dealing that characterizes decision-making within the intergovernmental legislative body of the European Union.

Before I delve in this response into the specific complaints by Heisenberg about the imagined and real limitations of rational choice theory and the Decision-making in the European Union project (DEU), I first position the Heisenberg chapter into the more general discussion in political science about the merits of formal models. To this end, I will distinguish between two possible ways in which we can read her chapter. Given my cultural background, I call these the 'Bismarck' and the 'Goethe' perspectives on the role of theory in political science. While the Bismarckian position advocates artful participatory insights rather than theory-driven empirical research as the way to understand politics, we should, according to Goethe's Mephisto in Faust I, privilege empirical experience over theoretical reasoning. As will become explicit in the following, I completely disagree with the first interpretation and would also like to qualify the second one severely. I will then dissect the Heisenberg argument, showing where she is definitively wrong and where she is at least half right. 
As the remainder will make clear, my main qualms with the preceding chapter are its mix-up between theoretical and empirical models and the apparent belief that rationalist scholarship necessarily needs to be tested in a quantitative fashion. I will also demonstrate that the 'culture of consensus' that Heisenberg advocates is in perfect line with the rationalist scholarship, as some of the extant bargaining models, which are barely mentioned, explicitly assume that actors have an inherent interest in cooperation. In contrast to Heisenberg's interpretation, bargaining models such as those presented in Thomson et al. (2006), Schneider (2005) or Schneider et al. (2006), have the distinct advantage that they provide clear and testable causal mechanisms. Hence, the competing evaluation of explicit, competing theoretical models, be they mathematical or verbal, is the backbone of the 'normal science' that I and many of my colleagues have been advocating since the mid-1990s and that, given Heisenberg's reaction, still provokes severe misunderstandings among traditional EU scholars. As this chapter will demonstrate, rationalist scholarship has contributed to our understanding of Council of Ministers decision-making so profoundly that both a Bismarckian and a Goethean counter-revolution would severely hamper scientific progress.

\section{Bismarck versus Goethe: how scientific and theoretical should we be?}

In a speech at the Reichstag in 1884 Chancellor Bismarck stated that 'Politics is not a science, like many professors fancy, but rather an art.' It would be naive to assume that such haughtiness has died out among politicians; what is, however, astounding is that many political scientists still have problems with the word 'science' in the designation of their discipline. Heisenberg's essay is, unfortunately, not completely free of the self-flagellistic rhetoric to which a considerable number of political scientists, especially supporters of the perestroika movement within the American Political Science Association (for Example, Monroe 2005), still subscribe. First of all, I do not see why we still need to juxtapose 'science' and 'politics' and to suggest that publications in top-tier journals - or at least those authored by what is perceived as the rationalist sect - do not contain any 'real-world' insights. On the contrary, as I will show further below, rationalists have come up with answers to the nine 'current questions of policy' listed by Heisenberg in her conclusion. Quite a number of these policy-relevant insights have been published in European Union Politics, the journal I have edited since its establishment in 2000 and the imagined editorial policies of which Heisenberg discusses. ${ }^{2}$ Heisenberg notes that the associate editors and I have written 'with satisfaction' that the field is moving into a more scientific tradition and that methodological rigour has gained ground. This remark obviously begs the question as to why happiness over the professionalization of an academic field should be 
a sin and what the alternative to methodological rigour would consist of: non-rigour, fortuitousness, or plain incompetence?

The turn to 'normal science' manifests itself in the increasing number of articles with systematic research designs and explicit hypotheses. It reflects, in my view, the need felt by many junior scholars (who, by now, might not be that 'junior' any longer!) throughout the past 15 years to move away from the inconclusive debates over unwieldy topics such as the uniqueness of the European Union. The continued escape into the argument that the EU is a special case and cannot be compared to other political systems leaves the impression that many colleagues still prefer to shy away from conducting systematic and thus comparative research. As any microbiologist would easily confess, every amoeba living on this planet is a sui generis being. However, this biological distinctiveness does not incite lab workers to be on first-name terms with all the amoebas they study, tracing their lives and deaths in details that are irrelevant for the question they are posing. Even if there were only one Council decision or amoeba to study, we could examine this case carefully and in a comparative fashion by building up convincing counterfactuals.

Although it might be hard for some diehard postmodernists to believe, even rationalists like myself start out on a research endeavour by posing such practical questions as who gains and loses in Council negotiations, and what are the effects of the deliberation in a particular committee? Like all scholars who try to explain the real-world differences between comparable cases, we need to seek convincing explanations for variances and a method that allows us to test our hypotheses against competing explanations. Any serious research question thus drives us to the development of a theoretical and an empirical model which are, and this needs to be stressed, independent of each other. There is, in other words, nothing inherent in rational choice models that would privilege quantitative testing strategies over qualitative ones, as the essay by Heisenberg suggests. Moreover, neither the topic under examination, the training we received as a student nor our preference for a particular approach should guide us in the selection of a research design. The decisive criterion should rather be the nature of the hypotheses that we derived from a theoretical model and that we would like to examine empirically.

Hence, we need to think carefully about the appropriateness of a method for the particular theoretical claim that we would like to advance. If a theory is very strong and leads to a deterministic hypothesis ('If $\mathrm{x}$, then always $\left.y^{\prime}\right)$, we can resort to a single-case study to falsify the theoretical claim. In the event that there are many competing explanations and the key claim is probabilistic, we simply need to avoid a situation with 'negative degrees of freedom' where the number of explanatory variables exceeds the number of cases. This means that parsimonious theories can be tested within a very small sample. However, if we possess information on the universe of cases, such as all legislative initiatives deliberated in the Council of Ministers, then it is unreasonable not to compare all these events. 
In most instances, we have to satisfy ourselves with a sample of cases. This is what I did in collaboration with colleagues from the Netherlands, Finland, the United States and Germany in the DEU project, the alleged failings of which Heisenberg extensively discusses. As she writes in this context, it is difficult to come up with measures that adequately reflect the complexities of Council decision-making. However, simply to conclude that behavioural output is not a measurable variable reflects a basic lack of confidence in what can be achieved within political science today. The DEU project and related research endeavours amply show that we can obtain reliable information when we ask independent experts what the positions of policymakers in a debate are and, later on, when the decision is made, the location of the final outcome. One obvious problem of such expert interviews is the need to buy into the assumption that the positions reflect to some extent the true preference of a state. As Bailer (2005) convincingly demonstrates, the DEU preferences stand, to a considerable extent, for objective socioeconomic characteristics of a member state and are thus not purely strategically chosen. Furthermore, the claim by Heisenberg that economists measure only objective or revealed preferences is simply wrong, as the trend toward using survey data in this neighbouring social scientific discipline easily confirms - not to mention such revolutionary endeavours as 'behavioural economics' and the attempt to synthesize rational or boundedly rational actor models with the results found in psychological experiments. It should further be noted that Heisenberg misconstrues the way in which we proceeded in the DEU project. In the Thompson et al. (2006) volume, all empirical chapters in which a class of models is tested contain case studies. However, as our conjectures are probabilistic, we use these cases only as illustrations and test our claims by contrasting all cases within our sample of EU decisions.

Heisenberg's reproach that formal models lead to quantitative tests is probably a consequence of an unfortunate mix-up between theoretical and empirical models. As indicated, we need to keep these separate to avoid bias and to encourage attempts to refute our findings. The refusal to engage with tests that are independent of the theoretical model ultimately leads to the development of irrefutable claims and unwieldy research designs, furthering the cynical Bismarckian conviction that political science is an art that cannot properly be tamed.

The more interesting and rewarding interpretation of the Heisenberg chapter follows her desire to see more and better empirical research on the Council of Ministers. I would like to second her in this ambition. Her complaint that formal theorists have often shied away from getting their hands dirty through empirical research and, even if they still engage in some testing, hardly find support for their supposedly grandiose claims has a long history. In his play Faust Johann Wolfgang von Goethe lets Mephisto express the view that any science without proper empirical foundation is bloodless: 'Grey, my dear friend, is all theory and green the golden tree of life.' 
Most formal theorists would agree that contributions to positive political science need some empirical backing which rigorously contrasts the equilibrium predictions with systematic evidence. This is, however, neither the case for normative theory, in which the welfare implications of some specific rules are tested against some clearly specified standard like the Pareto criterion, nor for axiomatically derived existence results. Hence, there is no need to 'test' a purely mathematical result such as Arrow's Impossibility Theorem, the arguably most celebrated non-existence statement in formal reasoning. Applied rational choice theory, however, makes claims about the 'real' world, and we need to test the competing claims, including informally advanced hypotheses such as the 'culture of consensus' thesis and the formally derived propositions examined in the DEU project, against each other.

In other words, comparative theory evaluation could easily be extended to theories other than those tested in the DEU monograph and related publications. The only requirement would be that the researchers advancing such alternative theoretical accounts translate their claims into a testable form. Simply to suggest that the DEU project has not led to any new insights fails to recognize what the goal of this multinational research project was. The major aspiration was not to advance new models but to establish the predictive accuracy of existing ones. Heisenberg's claim that we already knew that bargaining is important in Council thus misses its target, as we were comparing negotiation models against alternatives and establishing that bargaining models do better than these alternatives. For a proponent of bargaining models like myself, it is obviously nice to hear that this scientifically established result confirms the untested prior belief of Heisenberg that, if the Council moves, it does so through negotiations. Statements such as 'we knew this before', however, simply reflect the unfortunate Mephisto-like tendency to generalize from pure observation. An indication of Heisenberg's empiricist leaning is her favourable quote of Corbett (2001), who, according to her, gave evidence of the allegedly 'flawed' nature of the original spatial model of EU legislation. As has been shown amply elsewhere (for example, Sokal and Bricmont 1998), participatory observation has real limits if the insight is founded on the wrong theory. Hence, a participant might not be able to explain potential outcomes that are not happening or are rare events within a political game if his or her prior experience is that such things as dissent hardly ever matter. Inductivism is exactly also the problem of the metaphor that a 'culture of consensus' rules Council interaction. If we do not advance some causal mechanism as to why unanimous decisions are more frequent than one would expect we cannot move further in our understanding of this particular institution. Most trivially, the 'consensus' could simply be a consequence of the tendency of the Commission to introduce legislative proposals that make a majority of the member states happy. As can be shown fairly easily with a spatial model, if each and every one is far away from the status quo, radical and harmonious change is possible (Schneider 1997). Another 
possibility that Heisenberg does not discuss arises from the piecemeal way in which the European Union makes decisions. Hence, the Commission quite frequently simply refuses to initiate legislation to which a majority of the member states would object. If it does, however, dare to introduce a particularly controversial piece of legislation, the chances are great that it is dropped from the agenda early on. Controversial cases thus hardly ever reach the final stage of voting. Finally, as a broad rationalist literature originating in Taylor (1976) and Axelrod (1984) tells us, actors can become more consensual if they know that they are likely to meet more frequently in the future. In a purely formal article on the 'culture of consensus', Carruba and Volden (2001, p. 22) exploit this logic and 'predict consensus to be more common on the most "difficult", or, equivalently, least valuable, legislation'. This counter-intuitive result is in considerable contrast with those studies which treat consensus as a constant or as an assumption rather than a variable that ought to be explained.

\section{A consensus on the 'culture of consensus'?}

One of the most problematic aspects of Heisenberg's chapter above is the limitation within the argument of rational choice contributions to the spatial theory of voting and the voting power literature. The latter approach has played only a minor role in the empirical study of Council decision-making simply because the indices which reflect the a priori power or influence of a Council member do not lend themselves easily to systematic tests. They have mainly been used for the calculation of how powerful a state could be after an enlargement round or an institutional reform. Such ex ante calculations are, however, misleading in situations in which no Rawlsian 'veil of ignorance' is lifted and delegates from the member states know what they can expect from the other member states under a new regime. Collaborative research within a particular Council of Ministers, such as the round of the agricultural ministers, has shown that we can predict up to two out of three of the preference configurations (Zimmer et al. 2005). This simply means that we are forced to include preferences in our theories of Council decisionmaking. What is more, the preferences of incoming member states are easily anticipated by the current members so that we cannot even sensibly rely on such measures to assess the power or influence of the Council of Ministers prior to an enlargement with the help of voting power indices. ${ }^{3}$

The spatial theory of voting is one - but not the only - alternative to the voting power literature. The first contributions were by Steunenberg (1994) and Tsebelis (1994), correcting Heisenberg's claim that the formal modelling tradition in European integration studies has its origins in US political science. ${ }^{4}$ It is, indeed, correct to say, as Heisenberg does, that spatial models were only tested systematically some years after these groundbreaking contributions. But it is incorrect to claim that institutions in combination with 
preferences only have a marginal influence on Council outcomes. On the contrary, Drüner et al. (2007) show that one of the core concepts used in the spatial theory of voting - the winset - has a high predictive accuracy with regard to the reform capacity of the EU and the duration of decision-making processes. Within the DEU project, the predictive accuracy of the legislative models that were tested was, admittedly, quite weak. The important addition to this qualification is, however, once again that they performed relatively badly relative to other models. This relative failure does not make the entire research tradition superfluous as a low predictive accuracy of a model in one context does not necessarily invalidate the usefulness of this approach in other applications. To do so would be equivalent to throwing out an influential and costly weather prediction tool after its failure to predict local conditions in some communities at some time. Proper attempts to evaluate the predictive accuracy of a model refer to the average forecast error or related criteria and not to occasional aberrations that can also be observed in other contexts.

The relatively successful DEU models are all bargaining models, some of them adhering to the cooperative and a few adhering to the non-cooperative modelling tradition within game theory. In my view, bargaining models are exactly the tool that Heisenberg could rely on to substantiate her claim that a 'culture of consensus' governs Council interactions. Most of the formal and informal bargaining models of Council interactions are based on the assumption that a bargain has to be Pareto superior. Hence, all these already developed and potential models of Council interactions, be they based on the Nash Bargaining Solution, the Kalai-Smorodinsky Bargaining Solution or alternative concepts, postulate that a final negotiated outcome will be compromise. ${ }^{5}$ Even if we resort to a non-theoretical model and expect the outcome to be somewhere in the middle of the taken positions, we can assume that an implicit assumption about the cooperative motivations of the actors has been made by the proponent of such a 'causal mechanism'. In other words, we have different notions of what form a consensus could take and if we want to move the 'culture of consensus' from a post hoc observation to a theory with predictive capacity, we need to test which compromise model is the correct one. If we, however, continue to see the 'culture of consensus' as a constant, we can never uncover why we have more compromise in some contests and more dissent in others.

Unfortunately, Heisenberg is not explicit about what she understands as the 'culture of consensus' and what outcome would be predicted on the basis of her implicit bargaining model. The following illustration from a case examined within the DEU project shows that we can come up with very different forecasts of where a compromise is situated in EU legislative negotiations. Figure 15.1 illustrates for the three contested issues of the E-commerce directive how well different theoretically naive and sophisticated bargaining models are able to explain EU decision-making and that quite different 
Issue 1: Country of services

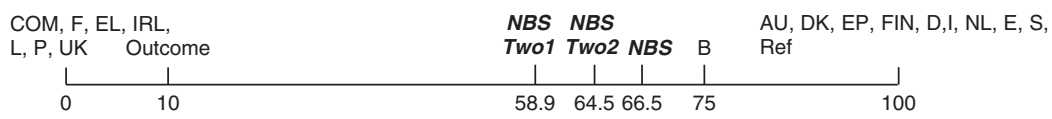

Issue 2: Inclusion of services
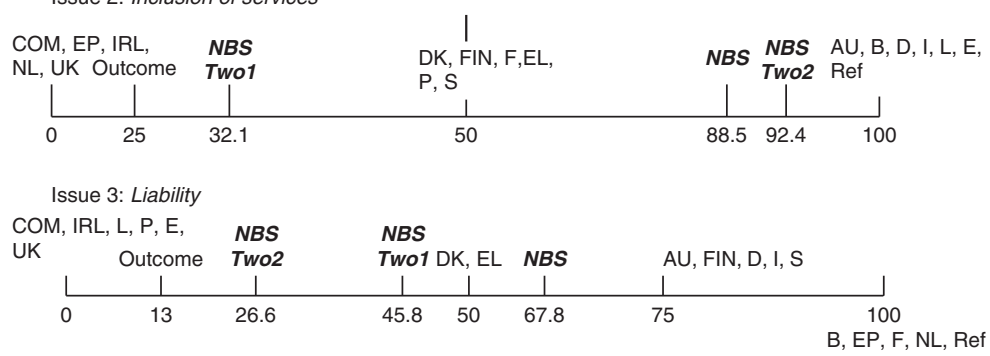

Figure 15.1 Decision-making on three contested issues within the E-Commerce proposal of the European Commission ${ }^{\mathrm{a}}$

Notes: a. I would like to thank Stefanie Bailer for allowing me to draw on our joint work in this figure. NBS stands for the prediction of the simple Nash Bargaining Solution, NBS-Two1 is a twolevel game version of it that considers also the power of the EU affairs committee in the member states' parliaments, and NBSTwo2 takes the EU affairs committee as well as its preferences into account.

Source: DEU data set and Bailer and Schneider (2006).

consensual solutions can be predicted. As in Bailer and Schneider (2006), I distinguish between three theoretical models - a simple Nash Bargaining Model (NBS), a NBS model which only considers the power of the EU affairs committee of the national parliaments and a third NBS model that again takes the power of the EU affairs committee as well as its policy preferences into account. The bargaining range goes from 0 to 100 .

The negotiations over the e-commerce directive show that the predictive accuracy of the competing consensus-oriented bargaining models can differ widely. While on the first issue all three predictions are quite far away from the outcome, the first two-level games offer the most precise forecast on the second issue and the second two-level game on the third issue. The illustrative evidence alerts us to the need to move beyond the 'culture of consensus'-metaphor and to explore under what conditions competing operationalizations of consensus do well in predicting real outcomes. The performance of the third institutionalist model shows again that we should not easily dismiss the importance of institutions as a constraint. The concluding chapter to the DEU volume advances the view that we should develop mixed models which 'combine a particular characterisation of an extensive form bargaining game with the procedural voting game played according to legal rules' (Schneider et al. 2006, p. 301). Hence, one particularly promising 
way to go in studies of the Council of Ministers is to resort as a first step to the spatial theory of voting, which helps us to detect the sub-set of potential outcomes of a decision-making process. Based on this information, we might want in a second step to resort to a standard bargaining model to predict a unique point as the result of EU legislation.

\section{The potential of rationalist scholarship}

This reply has already shown that Heisenberg has misrepresented the past achievements of the rationalist scholarship on the EU to a considerable extent. I will now demonstrate that she also underestimates the potential for future rationalist explanations of Council of Ministers decision-making. Interestingly, she lists in her conclusion a couple of research questions on which, in her opinion, rational choice institutionalists are 'fairly mute'. A proper reading of the rationalist literature would, on the contrary, have shown that exactly those questions have played a central role in rationalist scholarship so far and are likely to do so in the future. Taking the questions in turn.

\section{One-country, one-vote rule}

Heisenberg first poses the question of whether returning to unanimity would affect the decision-making capacity of the institution. Of course, such a counter-revolution would have undesirable effects, as many spatial models have shown. The puzzle of whether or not changing majority thresholds would affect the ability of the Council to act is a key consideration in many studies by applied formal theorists, most recently Steunenberg (2002), König and Bräuninger (2004) as well as Schneider et al. (2007). Technically, this literature explores whether the reform of the decision-making structure in the EU or the admission of new member states creates a danger of so-called gridlock. Drüner (2007) shows most convincingly that such fears have to be differentiated. While the 2004 and 2007 enlargements have increased the danger of inertia in decision-making, the risk of decision-making cycles has been further reduced. Heisenberg also asks whether a one country-one vote rule would increase the number of issue areas. The relationship between formal integration and the depth of integration is a standard topic in rationalist scholarship. The empirically supported model by Alesina and Spolaore (2003) suggests that more democracy and hence a move towards majority rule should lead to disintegration. This means that Heisenberg's suspicion that less ambition in constitutional matters could lead to more policy domains being integrated makes sense. However, a formal model by myself also suggest that the relationship between widening and deepening is more involved if, probably in line with Heisenberg, we move to a more complex model of political decision-making (Schneider 2002). 


\section{Giving up the rotating presidency}

The Treaty of Lisbon introduces three versions of the presidency: a full-time president of the European Council for renewable terms of two and a half years; the High Representative as the permanent chair of the Council dealing with external relations; and a version of the rotating presidency (in teams of three) in most other areas of Council business. Heisenberg asks the question of what would happen to negotiations in the absence of the traditional rotating presidency. Obviously, such a counterfactual is hard to bolster with solid empirical evidence. The problem is exacerbated by the relatively small number of models that address the power of the EU Presidency directly (Selck and Steunenberg 2004 for an exception). Fortunately, the computer simulations by Kollman (2003), which offer some protestant heresy in the rather catholic formal literature on EU decision-making, give some hints of what the advantages and disadvantages of a rotating presidency are. The mathematical evaluation shows that the collective utility of a rotating Presidency decreases when new member states join the Council. The rotating Presidency has, however, some collective advantages when the organization has to tackle a problem of medium-level difficulty. As Kollman (2003, p. 71) writes, 'holding constant the number of members of the Council, the relative advantage of the rotating presidents procedure decreases and then decreases as the difficulty of the policy problem increases'.

\section{The role of COREPER}

I agree with Heisenberg that the Committee of Permanent Representatives is one of the most understudied bodies within the European Union. However, there is some emerging evidence that the preparatory meetings of the member state delegates are not influenced by socialization but rather by institutional considerations (Häge 2007). Hence, also the preparatory meetings of Council decision-making can be explained through the negotiation perspective that is advanced in this chapter.

\section{Old versus new member states}

It is still too soon fully to assess how the new member states behave in the enlarged Council of Ministers, although several chapters in this volume include such data (Mattila, Hagemann, Naurin and Lindahl and Thomson). Hagemann notes that no 'new versus old' bloc is apparent, while Naurin and Lindahl in chapter 4 claim to have found a new Eastern dimension in the cooperation patterns after enlargement. Using the DEU data set and additional interviews, however, my collaborators and I addressed this question in 2004. The answer is that whether we can expect the new member states to behave differently from the old member states depends on the policy area (Dobbins et al. 2004). No member state is strong enough to block the organization in the long run on its own, and, hence, coalition-building remains the key to advancing one's own position in the Council. 


\section{Change in decision-making styles}

Heisenberg asks how we would know that decision-making styles have changed. Indeed, I do not know any systematic study that traces the usage of particular negotiation tactic over time. Bailer's $(2004,2005)$ cross-sectional evidence, however, suggests that the way in which states behave largely reflects their attractiveness as a coalition partners. Such a study would have to be replicated in order to provide solid evidence over changes in behavioural patterns over time. Some preliminary evidence that the past two enlargements might have changed the interactions in the Council comes from Hagemann in this volume and Hageman and De Clerck-Sachsse (2007b). They show, based on elite interviews, that member states more frequently resort to formal statements to make their point. This enables government 'to affect a sense of the old culture of consensus without at the same time sending a political signal of having deviated from their initial policy preferences' (Hageman and De Clerck-Sachsse 2007b, p. 14). It remains to be seen whether such changes have a more permanent nature

\section{Enhanced cooperation}

There has been an intensive normative discussion of whether or not the European Union has reached its 'limits to growth' (for instance Alesina and Spolaore 2003). The related tension between widening and deepening has been one of the key considerations in rationalist scholarship. While some of the models address this trade-off through the lenses of club good theory (for instance Sandler and Tschirhart 1997), others also invoke the costs of decision-making (for instance Schneider 2002) or of increasing social heterogeneity (for instance Alesina and Spolaore 2003) with which an expanding organization has to reckon. This literature also discusses the need to move to more flexible institutional arrangements which are studied from a rationalist vantage point in Kölliker (2005).

\section{Dominance of member states}

One of the dangers for any federation is that one state is able to profit more from the organization than other member states. The DEU project has explicitly addressed this topic and established that that there are no absolute winners in Council of Ministers decision-making across all policy sectors (for instance Bailer 2004), but that a logic of redistribution governs many interactions of this intergovernmental body (Zimmer et al. 2005). The absence of long-term winners in EU legislation could be one of the ingredients for the success of the organization, but we do not possess sufficient systematic information on vote trading beyond sectoral limitations.

\section{Conflicting preferences}

We have little empirical knowledge on how 'council representatives interface with national parliaments when preferences conflict'. However, this question 
has played an important role in the principal-agent literature that has come to dominate the study of delegation in the EU (Franchino 2007). The groundbreaking theoretical and empirical work by Franchino exactly points out the conditions under which member states prefer to delegate legislative work to the Commission or to the national parliaments. Moreover, Pahre (1997) and Martin (2000) show how executives can use domestic opposition to bolster the credibility of their demands.

\section{Leeway}

Interestingly, Heisenberg takes up the Schelling conjecture or the so-called paradox of weakness in her list of unanswered questions about Council of Ministers' decision-making and asks whether constrained member states are more powerful than less constrained ones. This is exactly the topic that Bailer and Schneider (2006) address in the DEU volume. Based on the twolevel game specifications of the NBS model briefly sketched in this article, they reject this important theoretical insight for this decision-making arena, noting that there are ample reasons to suspect that European Council deliberations follow the Schelling logic to a much greater degree than interactions among the Council of Ministers (Schneider and Cederman 1994).

\section{Conclusion}

This chapter has discussed some of the objections by Dorothée Heisenberg to recent rational choice scholarship on the Council of Ministers. While I have taken issue with some of her anti-rationalist statements, I would - once again - point out that I fully agree with her that we need better models and empirical tests. My US colleague and I, to paraphrase Nobel laureate R. Aumann, also agree to disagree on how we can achieve this lofty goal. In my view, game-theoretic models will play a key role in the future study of the Council of Ministers as their underlying assumption of strategic rationality is well-justified in a world in which elected politicians are forced to represent the interests of their voters at least to some extent and where fiscal temptations often loom large (Zimmer et al. 2005). In contrast to Heisenberg, I welcome the turn towards 'normal science' in EU studies. This development has allowed the discipline to engage in the comparative evaluation of competing theoretical models. It has particularly encouraged the research community to assess what sort of model most accurately predicts the outcomes of Council interactions (Thomson et al. 2006), who the winners and losers of EU decision-making are (Bailer 2004) and whether Council deliberations follow the logic of arguing or bargaining (Franchino 2007).

Obviously, such 'normal science' might be dull at first sight and can certainly not cover all the complexities of the Council decision-making; but it is not its aspiration to explain all trivialities, technicalities and banalities. Normal science, like any good science, needs patience - the fortitude to develop 
convincing causal mechanism and the endurance in making sense out of empirical reality. If the insights of rationalist scholarship on the Council are trivial, as Heisenberg implies, I have no problem with this because, post hoc, many great and ground-breaking contributions seem small. What is needed is not simple, it is major innovation and risk-taking behaviour that takes us beyond the easy consensus - like the one on the 'culture of consensus'.

In achieving the goal of systematic thinking about the interactions within the Council of Ministers, we can neither follow the Bismarckian interpretation ('political science is an art') nor the Goethean one which privileges experience over theory. We should rather heed the advice of Cole Porter (Porter and Kimball 1983) who, jokingly, called for a (quasi)-experimental approach that will lead us 'to the light'. In my view, the best way to move beyond inconclusive debates about whether qualitative or quantitative work is better in explaining Council decision-making or whether the EU is a sui generis being we should simply ask questions that are answerable, develop convincing causal mechanisms and then test them. If we all follow this advice, we will surely no longer debate whether formal models have made any contribution, but will rather ask which model, formal or informal, delivers the best predictions in a particular context. In other words, we need a culture of dissent to understand the imagined or real 'culture of consensus' that allegedly guides Council interactions.

\section{Notes}

1. I would like to thank the editors, Stefanie Bailer, Daniel Finke, and Robert Thompson for comments on an earlier draft of this article.

2. Interestingly, Heisenberg is not the only scholar who commented upon the two editorials that Matthew Gabel, Simon Hix and I published in 2000 and 2002. Manner's (2007, p. 90) criticism is particularly pointed. According to him, critical perspectives 'offer the opportunity to escape the normative wasteland and monstrous claims of "normal science" of economist pathologies - that path only leads to tighter straightjackets [sic].'

3. I note the recent attempts by Widgrén and colleagues to integrate preferences into the voting indices. Previous attempts to do so have been hampered by the sensitivity of the model predictions to small variations in the preference assumptions. Whether the new approach has overcome this problem is, to my knowledge, not yet decided. For a more philosophically grounded debate on the new models see Braham and Holler (2005) and Napel and Widgrén (2005).

4. The same year also saw the publication of formal models that were derived at least partly by Europeans, as for instance the predecessor to the DEU research project volume (Bueno de Mesquita and Stokman 1994) and the non-cooperative bargaining models of European Council deliberations by Schneider and Cederman (1994).

5. Butler (2004) and Muthoo (2000) offer accessible introductions to the bargaining literature. See also Muthoo (1999) for a technical introduction and Schneider (2005) for a discussion of how different forms of power may influence the outcome of consensual bargaining. 